\title{
Sepsis and meningoencephalitis due to Rhodotorula glutinis in a patient with systemic lupus erythematosus, diagnosed at autopsy
}

\author{
Umabala Pamidimukkala, Sundaram Challa*, Vemu Lakshmi, Ashwani Tandon*, Srinivas Kulkarni**, \\ Satyanarayana Y. Raju** \\ Departments of Microbiology, *Pathology and **Medicine, Nizam's Institute of Medical Sciences, Hyderabad, Andhra Pradesh, India
}

\begin{abstract}
Rhodotorula species have been reported as a causative agent of opportunistic mycoses in immunocompromised hosts. We report a case of sepsis and meningoencephalitis caused by Rhodotorula glutinis in a 20 -year-old female patient with systemic lupus erythematosus (SLE), which was diagnosed at autopsy. The patient presented with longstanding fever. She was diagnosed with SLE after admission to the hospital and died on day 5 of the hospital stay. Autopsy was performed to confirm the presence of infection. Sepsis and meningoencephalitis due to Rhodotorula glutinis was confirmed by postmortem blood cultures and histopathological examination of biopsies taken from the brain at autopsy. Infection by Rhodotorula spp. is rare but can be fatal in immunocompromised hosts. Infections by such uncommon yeasts may often be difficult to diagnose, especially in the setting of febrile neutropenia. This report also emphasizes the value of autopsy as a powerful educational tool.
\end{abstract}

Key words: Autopsy, fungemia, meningoencephalitis, postmortem blood culture, Rhodotorula, SLE

\section{Introduction}

The yeast rhodotorula, a member of the family Cryptococcaceae, is a common airborne fungus found in the skin, lungs, urine and feces. Like other yeasts and yeast-like organisms, rhodotorula species has been previously considered a nonvirulent saprophyte; although in recent times, cases have been reported of both local and systemic opportunistic infections by these yeasts. ${ }^{[1-3]}$

\section{Case Report}

A 20-year-old female patient was admitted on $11^{\text {th }}$ September 2003 to a tertiary care hospital in Hyderabad, Andhra Pradesh, India, with a history of high-grade continuous fever, not associated with chills and rigor, since 2 months. The patient also complained of cough, bleeding gums, loss of weight, body pains and amenorrhea since 2 months. On general examination, the patient was found to be febrile, malnourished and cachectic. Marked pallor was present, along with mild splenomegaly, cervical and axillary lymphadenopathy. With a clinical picture of pyrexia of unknown origin, the patient was investigated further.

Blood cultures were done using the Automated BacT/ Alert (bioMerieux, France) continuous-monitoring blood culture system. One of the two blood cultures drawn on day 1 of hospital stay showed growth of coagulasenegative staphylococci after $26.2 \mathrm{~h}$ of incubation; and the isolate was reported as a probable contaminant from the skin, with advice to repeat the culture. The remaining bottle was sterile after 5 days' incubation. Hematological examination performed on day 1 revealed anemia (Hb: $7.7 \mathrm{~g} / \mathrm{dl}$ ), thrombocytopenia (platelet count: $80,000 / \mu \mathrm{l}$ ), mild leukopenia (total leukocyte count: $3.1 \times 10^{9}$ ). Peripheral blood smear for malarial parasite and febrile agglutination tests were all negative. Antinuclear antibodies were reported positive, along with antibodies to extractable nuclear antigens, dsdna and sm - suggesting a diagnosis of SLE. The direct coombs test was positive, while indirect coombs test was negative. Bone marrow examination showed a picture of lymphoplasmacytosis. The patient's condition deteriorated in spite of treatment, and she expired on day 5 of the hospital stay.

Consent was taken for complete autopsy, which was performed within $2 \mathrm{~h}$ of death. Standard protocol for whole body autopsy was followed. Postmortem blood cultures were drawn prior to any other manipulation of either thoracic or abdominal organs. Ten milliliters of blood was drawn from the right side of the heart with a sterile syringe and needle, after disinfecting the surface with spirit and $7.5 \%$ povidone iodine. Five milliliters 
each was inoculated into two BacT/Alert blood culture bottles. Another BacT/Alert bottle was inoculated with $5 \mathrm{ml}$ of blood obtained aseptically with a separate syringe and needle by splenic puncture. All three bottles were incubated in the BacT/Alert blood culture system. All the organs were dissected and processed for histopathological examination as per standard protocols. The brain and the upper part of the spinal cord were fixed in $10 \%$ buffered formalin.

\section{Postmortem microbiology results}

All three BacT/Alert blood culture bottles, inoculated with heart blood and splenic blood, were determined positive within 2.9-5 days after incubation. Gramstained smear of the blood culture broth showed globose yeast cells without any pseudohyphae. Subcultures done on Sabouraud's dextrose agar from the bottles showed growth of soft mucoid orange colonies within $24 \mathrm{~h}$. The isolates were identified as Rhodotorula species by the colony morphology [Figure 1] and microscopic appearance [Figure 2]. Further speciation of the isolate as Rhodotorula glutinis was done using the API ID 32C yeast identification strip (bioMerieux, France). Other specimens collected at autopsy - viz., CSF, peritoneal fluid, bone marrow - did not show any bacterial or fungal growth.

Antifungal susceptibility testing was done using the ATB Fungus 2 (bioMerieux, France). Amphotericin B (MIC: $<0.5 \mathrm{mg} / \mathrm{L}$ ) and flucytosine (MIC: $<0.5 \mathrm{mg} / \mathrm{L}$ ) were both active in vitro. The strain was resistant in vitro to fluconazole (MIC: $64 \mathrm{mg} / \mathrm{dl}$ ) and itraconazole (MIC: $>4 \mathrm{mg} / \mathrm{dl}$ ).

\section{Pathological findings}

Gross examination of the external surface of the brain showed hazy meninges [Figure 3]. There were no basal exudates, and vessels forming the circle of Willis were unremarkable. Ventricles were of normal size. Cerebellum and brain stem were unremarkable. All the lobes of the right lung showed hemorrhagic infarcts. Liver showed a superficial hematoma. Rest of the organs were normal. Histopathological examination of sections from the brain revealed congested vessels and minimal lymphomononuclear infiltrates in the subarachnoid space [Figure 4] and at the ventricular surface. Gomori's Methenamine Silver (GMS) staining of the sections from the brain showed round-to-oval budding yeast forms of varying sizes in the subarachnoid space [Figure 5] and at the ventricular surface [Figure 6].

\section{Discussion}

Members of the genus rhodotorula are ubiquitous in distribution. It is a yeast found in air, soil, lakes, ocean water and dairy products. ${ }^{[1]}$ It may colonize plants; and in humans, it has been isolated from feces, skin, sputum and digestive tract forming part of the normal flora. ${ }^{[4]}$

The genus rhodotorula is a pigmented yeast classified under the family Cryptococcaceae and includes 38 species. ${ }^{[5]}$ The species most commonly isolated from clinical specimens are: R. mucilaginosa (previously named Rhodotorula rubra), R. glutinis, R. minuta. ${ }^{[3]}$ Rhodotorula species has several morphologic and physiologic similarities with Cryptococcus species but differs from it by the typical carotenoid pigment and by the inability to assimilate inositol. ${ }^{[6]}$

Lunardi et al. ${ }^{[3]}$ reviewed 59 cases of rhodotorula blood stream infections during the period 1960-2003 and found that the major risk factor for rhodotorula fungemia has been prolonged use of indwelling intravenous catheters in patients with hematological and solid organ malignancies who are taking corticosteroids and cytotoxic drugs. In addition, use of broad spectrum antibiotics has been consistently reported in patients with rhodotorula fungemia. Anaissie et al. ${ }^{[2]}$ found that persistent neutropenia and disseminated visceral infection correlated with poor prognosis in patients with rhodotorula fungemia. Our patient was diagnosed to be suffering from SLE in the present admission and did not receive any steroids prior to admission. Though the susceptibility to infections increases in patients with SLE due to the immunosuppressive treatment modalities, infections are common in these patients even in the absence of immunosuppressive therapy. Many abnormalities, including immunoglobulin deficiencies, acquired and inherited complement deficiencies, defects in chemotaxis, phagocytic activity and delayed hypersensitivity, may account for this susceptibility to infection. ${ }^{[7]}$ Other probable risk factors in our patient could be the prolonged antibiotic therapy prior to the present admission, severe malnutrition and leucopenia.

Rhodotorula species have been implicated as a cause of meningitis, endocarditis, ventriculitis, peritonitis, fungemia, central venous catheter infection and keratitis. $^{[1,2]}$

In the present case, there was histopathological evidence of involvement of the meninges and brain parenchyma, in addition to the blood stream infection. After reviewing the literature on rhodotorula infections, the authors did not come across any reports of brain involvement though several cases of meningitis have been documented.

The present case report also emphasizes the need for a properly performed and documented autopsy to provide factual and scientific evidence for resolving medical questions. The indication for postmortem blood culture in this case can be categorized as class 1 as per the Cumitech, ${ }^{[8]}$ wherein postmortem blood culture is done to confirm the presence of an infection suspected clinically but could not be confirmed antemortem.

Rhodotorula infections have been successfully 


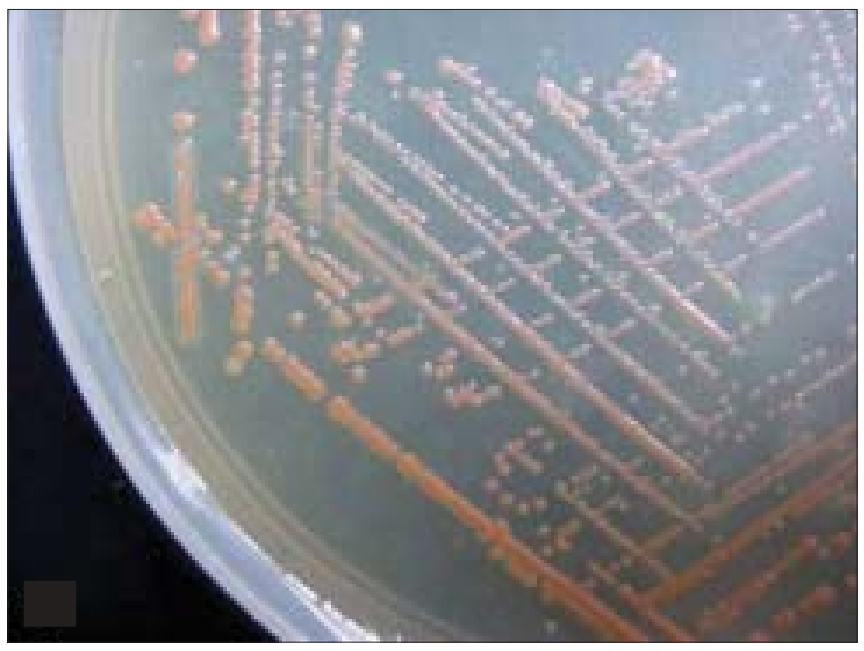

Figure 1: Soft mucoid orange colonies of rhodotorula glutinis on Sabouraud's dextrose agar after $\mathbf{4 8}$ hours' incubation

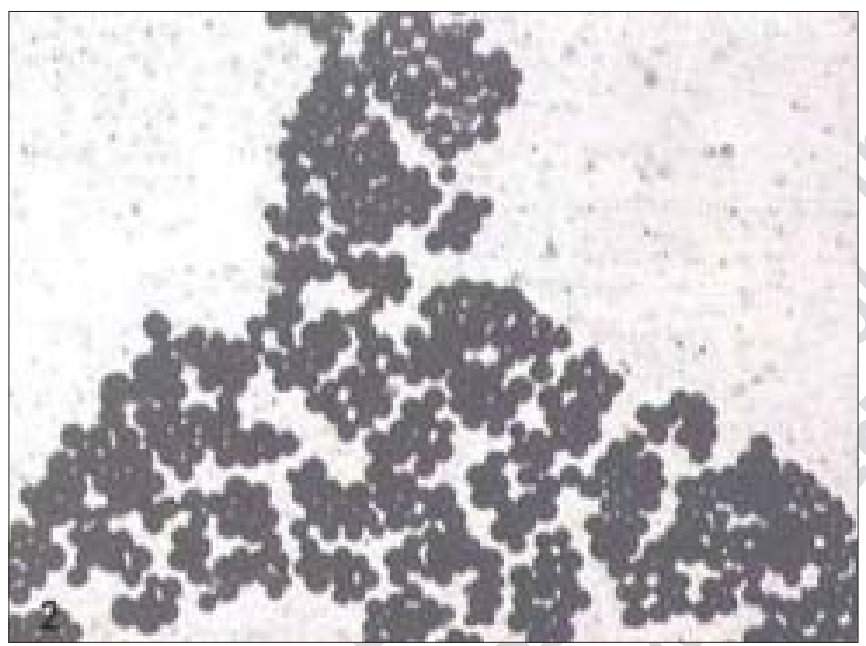

Figure 2: Smear from culture, showing spherical budding yeast cells of rhodotorula glutinis (Gram's stain, $\mathbf{x} 400$ )

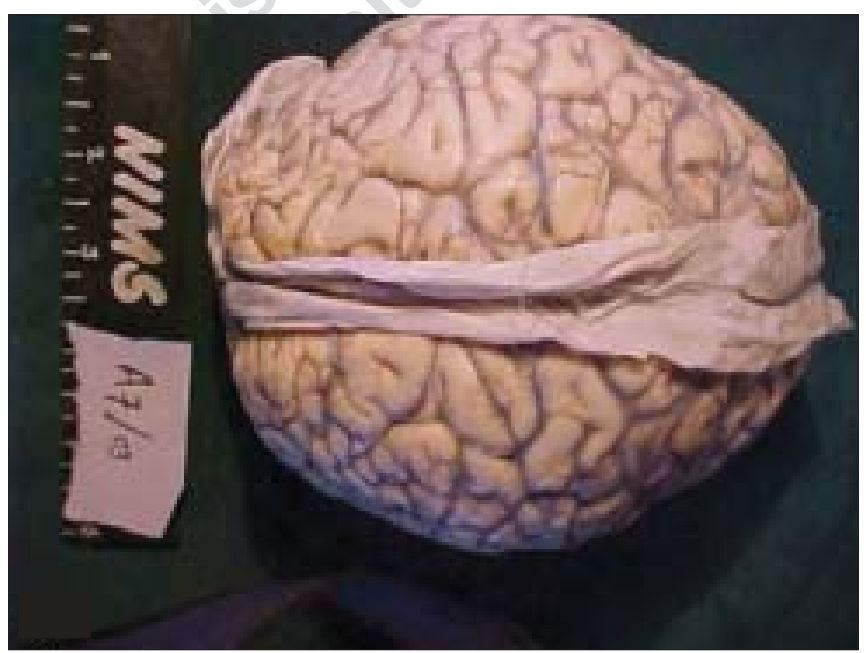

Figure 3: Superior surface of the brain showing haziness of the meninges with obliteration of sulci and flattened gyri

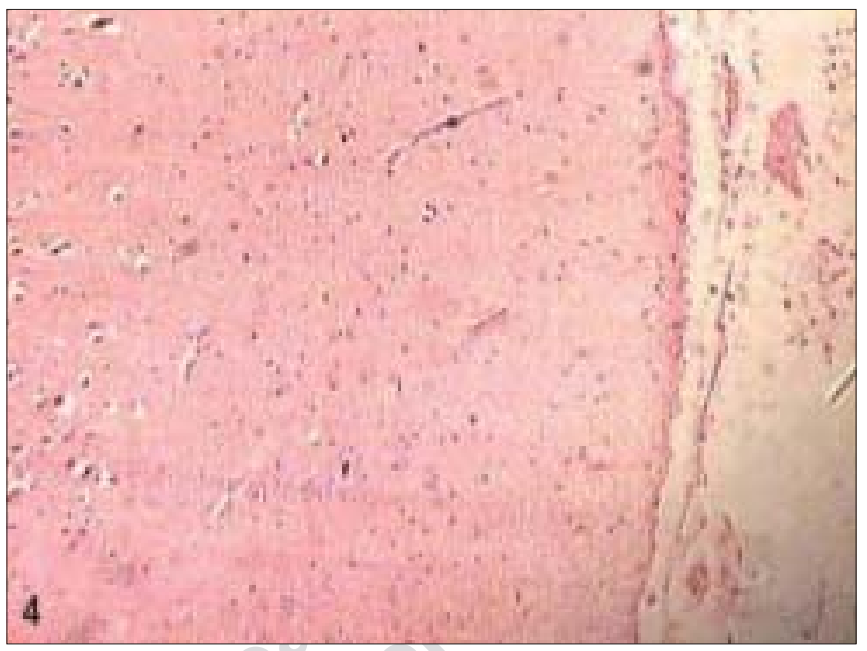

Figure 4: Meningeal surface showing congested vessels with minimal inflammation (H\&E stain, $\mathbf{x 4 0 )}$

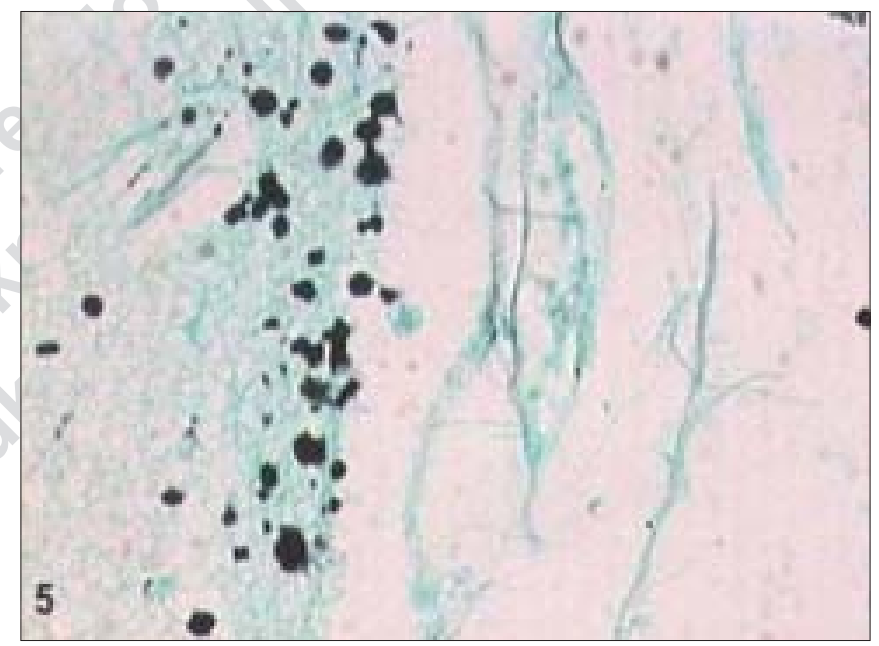

Figure 5: Meningeal surface showing budding yeasts of varying sizes (Gomori's Methenamine Silver stain, x400)

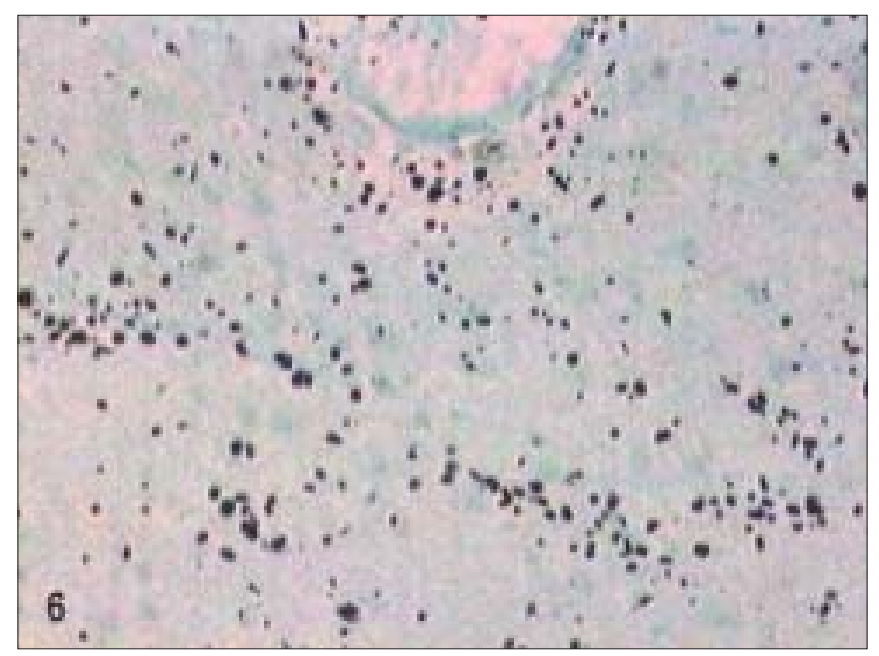

Figure 6: Ventricular surface and brain parenchyma showing budding yeasts (Gomori's Methenamine Silver stain, x100) 
managed with intra-vascular catheter removal alone, antifungal therapy without catheter removal and with a combination of these approaches. ${ }^{[3,9]}$ There are very few studies on the antifungal susceptibility of the rhodotorula species. Galan-sanchez et al. ${ }^{[4]}$ have tested 35 different strains of Rhodotorula for their susceptibility to different antifungals and have concluded that all antifungals except fluconazole are useful for the treatment of infections by rhodotorula spp. In a recent study, Zaas et al. ${ }^{[10]}$ determined the susceptibility of 10 rhodotorula blood stream infection isolates. Of the isolates tested, 8 were rhodotorula mucilaginosa and 2 were of the species rhodotorula glutinis. Two patients treated successfully with fluconazole had isolation of rhodotorula mucilaginosa and rhodotorula glutinis, which grew poorly at $35^{\circ} \mathrm{C}$ in vitro. The MIC of fluconazole used in these cases was high, $32 \mu \mathrm{g} / \mathrm{ml}$, but successful treatment with an antifungal agent demonstrating an elevated MIC was attributed to catheter removal alone or to decreased hardiness of the temperature-sensitive phenotype. The authors conclude that amphotericin B preparations, in addition to catheter removal, are acceptable therapies for rhodotorula infection, with excellent in vitro activity and reports of successful use. Flucytosine also was found to have excellent activity in vitro. Based on the in vitro data, echincandins and the narrow-spectrum azoles such as fluconazole were not considered appropriate therapy. The authors opine that further studies are needed to determine the role of extended-spectrum azoles such as voriconazole and posaconazole, given their wide spectrum of activity against Rhodotorula species. Diekema et al. ${ }^{[9]}$ reported the in vitro activities of eight antifungals against 64 rhodotorula isolates. They found that rhodotorula strains are resistant in vitro to fluconazole and caspofungin; amphotericin B and flucytosine are both active in vitro; and the newer broad-spectrum traizoles - voriconazole, posaconazole and ravuconazole - all have some in vitro activity, with ravuconazole being the most active. The results of antifungal susceptibility of the rhodotorula strain isolated from the present case correlate with the findings of the above studies.

In conclusion, immunocompromised patients, especially those with hematological and solid malignancies who are receiving broad-spectrum antibiotics, corticosteroids or cytotoxic drugs and who have long-term indwelling intravenous catheters, are susceptible to disseminated infections due to rhodotorula species. Infection by these rare yeasts could be under-reported because of difficulties in diagnosis in the setting of febrile pancytopenia and the danger of attributing isolates to specimen contamination. This case report also emphasizes the value of a properly performed autopsy and postmortem microbial cultures as valuable educational tools.

\section{References}

1. De Hoog GS, Guarro J, Gene J, Figueras MJ, editor. Atlas of clinical fungi. $2^{\text {nd }}$ ed. ASM Press: Washington, DC; 2001.

2. Anaissie E, Bodey GP, Kantairijian H, Ro J, Vartivavian SE, Hopfer $\mathrm{R}$, et al. New spectrum of fungal infections in patients with cancer. Rev Infect Dis 1989;11:369-78.

3. Lunardi LW, Aquino VR, Zimerman RA, Goldani LZ. Epidemiology and Outcome of rhodotorula Fungemia in a tertiary care hospital. Clin Infect Dis 2006;43:e60-3.

Galán-Sánchez F, García-Martos P, Rodríguez-Ramos C, MarínCasanova P, Mira-Gutiérrez J. Microbiological characteristies and susceptibility patterns of strains of R hodotorula isolated from clinical samples. Mycopathlogia 1999;145:109-12.

Chapter 5; Characteristics of the genera. In: Barnett JA, Payne RW, Yarrow D, Barnett L, editors. Yeasts: Characteristies and Identification, $3^{\text {rd }}$ ed. Cambridge University Press: 2000.

6. El-Tahawy AT, Khalef RM. R hodotorula rubra fungemia in an immunocompromised host. Ann Saudi Med 1999;19:533-5.

7. Ginzler EM. Infections in SLE. In: Dubois' Lupus erythrematosus. Wallace DJ, Hahn BJ, Franciseo P, Quismorio J, Klinenberg JR, editors. Williams and Wilkins Pub: 1997. p. 903-14.

8. Caplan MJ, Koontz FP, editors. Cumitech 35, Postmortem microbiology. ASM Press: Washington, DC; 2001.

9. Diekema DJ, Petroelje B, Messer SA, Hollis RJ, Pfaller MA. Activities of available and investigational antifungal agents against $R$ hodotorula species. J Clin Microbiol 2005;43:476-8.

10. Zaas AK, Boyce M, Schell W, Lodge BA, Miller JL, Perfect JR. Risk of fungemia due to R hodotorula and antifungal susceptibility testing of Rhodotorula isolates. J Clin Microbiol 2003;41:5233-5.

Accepted on 10-09-2007

Source of Support: Nil, Conflict of Interest: None declared. 